\title{
Identification and Expression Analysis of Histone Modification Gene (HM) Family During Somatic Embryogenesis of Oil Palm
}

\section{Lixia Zhou ( $\sim$ glzz_2009@163.com )}

Chinese Academy of Tropical Agricultural Sciences

\section{Rajesh Yarra}

Chinese Academy of Tropical Agricultural Sciences

\section{Longfei Jin}

Chinese Academy of Tropical Agricultural Sciences

\section{Yaodong Yang}

Chinese Academy of Tropical Agricultural Sciences

\section{Hongxing Cao}

Chinese Academy of Tropical Agricultural Sciences

\section{Zhihao Zhao}

Chinese Academy of Tropical Agricultural Sciences

\section{Research Article}

Keywords: HMs, Somatic embryogenesis, Oil palm, Real-time PCR

Posted Date: August 13th, 2021

DOl: https://doi.org/10.21203/rs.3.rs-785720/v1

License: (1) This work is licensed under a Creative Commons Attribution 4.0 International License.

Read Full License

Version of Record: A version of this preprint was published at BMC Genomics on January 5th, 2022. See the published version at https://doi.org/10.1186/s12864-021-08245-2. 


\section{Abstract}

\section{Background}

Oil palm (Elaeis guineensis, Jacq.) is an important vegetable oil yielding plant. Somatic embryogenesis is the promising method to produce large scale elite clones to meet the demand of palm oil. The epigenetic mechanisms such as histone modifications have emerged as critical factors during somatic embryogenesis. These histone modifications are associated with the regulation of various genes controlling the somatic embryogenesis. To date, none of the information is available on the histone modification gene (HM) family in oil palm

\section{Results}

We reported the identification of 109 HM gene family members including 48 HMTs, 27 HDMs, 13 HATs, and 21 HDACs in the oil palm genome. Gene structural and motif analysis of EgHMs showed varied exonintron organization and with conserved motifs among them. The identified $109 \mathrm{EgHMs}$ were distributed unevenly across 16 chromosomes and displayed tandem duplication in oil palm genome. Furthermore, relative expression analysis showed the differential expressional pattern of 99 candidate EgHM genes at different stages (non-embryogenic, embryogenic, somatic embryo) of somatic embryogenesis process in oil palm, suggesting the EgHMs play vital roles in somatic embryogenesis. Our study laid a foundation to understand the regulatory roles of several EgHM genes during somatic embryogenesis.

\section{Conclusions}

A total of 109 histone modification gene family members were identified in the oil palm genome via genome wide analysis. The present study provide an insightful information regarding $H M$ gene's structure, their distribution, duplication in oil palm genome and also their evolutionary relationship with other HM gene family members in Arabidopsis and rice. Finally, our study provided an essential role of oil palm $H M$ genes during somatic embryogenesis process.

\section{Background}

Histone modifications gene family (HMs) play a vital role in plant growth and developmental processes by histone modification processes (methylation, demethylation, acetylation, and deacetylation) either by activating or silencing the gene expression. These four different histone modification processes are regulated by four different $\mathrm{HM}$ gene family members such as HMTs (histone methyltransferases), HDMs (histone demethylases), HATs (histone acetylases), and HDACs (histone deacetylases) [1, 2]. Each of the $\mathrm{HM}$ gene family members also consisted of a varied number of subfamilies with different domain structures. The HMTs contained SDG (set domain group) and PRMT (protein arginine methyltransferases) sub-families. The HDMs contained HDMA (SWIRM and C-terminal domain) and JMJ (JmjC domain-containing proteins) sub-families. The HATs contained HAG (GCN5-, ELP3-, and HAT1-like histone acetylases domain structure), HAM (MOZ-YBF2 domain), HAC (p300/CREB-binding protein 
structure), and HAF (TATA-binding protein-associated factors $\mathrm{TAF}_{\|} 250$ ) sub-families. The HDACs contained HAD (RPD3/HDA1 superfamily), SRT (silent information regulator 2), and HDT (HD2 families) sub-families $[3,4,5,6,7$. Numerous previous studies described the role of $\mathrm{HM}$ gene family members in regulating the vegetative and reproductive growth, biotic and abiotic stress responses, stress-related hormone signaling $[4,8,9,10,11,12,13,14,15,16]$. Various genome-wide studies revealed the occurrence of a varied number of $H M$ gene family members in various plants including a total of $198 \mathrm{HMs}$ in Malus domestica[8]; 125 HMs in Lycopersicum esculentum[17]; 136 HMs in Citrus sinensis[18]; 87 HMs in Litchi chinensis [9]. However, to date, none of the studies were published on genome-wide studies of HM gene family members in oil palm genome.

African oil palm (Elaeis guineensis, Jacq.) is the most promising and productive oil crop to accomplish the increasing demand for vegetable oils around the world[19, 20]. The vegetative propagation for largescale production of oil palm plants is not possible due to the absence of auxillary shoots and traditional seed propagation is not much successful due to the low seed germination rate. Moreover, genetic improvement of oil palm plants via seed propagation is the most complicated. A promising substitute for the large-scale production of oil palm seedlings is the extensive micropropagation of plants via tissue culture method of approach i.e. somatic embryogenesis (SE). In plants, somatic embryogenesis is the method of producing somatic embryos under in vitro conditions without the fusion of the gametes [21]. Somatic embryogenesis is the reliable and powerful biotechnological approach for the micropropagation of plants with low seed germination rates as well as long reproductive cycles [22]. However, the somatic embryogenesis response is differed from species to species depending on their totipotency capacity. Though somatic embryogenesis protocols were well established for the propagation of oil palm, it's essential to understand metabolic, genetic, epigenetic, morphogenetic factors that boost the somatic embryogenesis process in oil palm plants. Moreover, epigenetic mechanisms such as methylation, demethylation, acetylation, and deacetylation are regulating the gene expression that modulates the capacity of somatic embryogenesis during tissue culture [23]. The epigenetic mechanisms that regulate the gene expression at somatic embryogenesis have not been addressed much in oil palm. Identification of genes that are responsible for regulating the epigenetic mechanisms during somatic embryogenesis of oil palm is vital for a better understanding of SE process. Up to now, none of the studies were reported on histone modification $(H M)$ gene family members in oil palm during different somatic embryogenesis phases.

In this study, we identified a total of 109 HM gene family members (48 HMTs; 27 HDMs; 13 HATs, and 21 $H D A C s$ ) in the oil palm genome via the bioinformatics approach. We also analyzed the EgHMs gene structure, motif analysis, phylogenetic analysis, synteny, promoter analysis, subcellular localization, and their location on 16 chromosomes in oil palm genome. Further, we analyzed the expression patterns of all identified EgHMs at different stages of the somatic embryogenesis process of oil palm.

\section{Material And Methods:}


The pfam database was used to identify the EgHM gene family members using the Hidden Markov Model profiles of published IDs of each type (HMT, HDM, HAT \& HDAC) of HM genes [17,18. The pfam IDs belongs each type of $H M$ genes were used as a query to search for $\mathrm{HM}$ gene members in oil palm genome database using HMMER3.0 tool. Further, we also retrieved the unavailable sequences of EgHMs using the known HM gene sequences of Arabidopsis (http://www.arabidopsis.org/) and Oryza sativa (http://rice.plantbiology.msu.edu/) through blast search in oil palm genome database (http://palmxplore.mpob.gov.my/palmXplore/).

We also predicted the coding sequence length of each $\mathrm{HM}$ gene family member using Blastn search against oil palm genome database. The identified putative oil palm HMs genes, such as HMTs (SDGs and PRMTs), HDMs (HDMAs and JMJs), HATs (HAGs, HAMs, HACs, and HAFs), and HDACs (HDAs, SRTs, and HDTs) were identified finally based on the highly conserved domains. Additionally, the M.wt, pl values of oil palm HM gene members were also determined with the help of ExPASy (https:// web. expasy. org/ compu te_ pi/). The online tool "CELLO" (http://cello. life. nctu. edu. tw/) was further used to predict the subcellular localization of all oil palm HM gene family members.

\section{Gene structure, Conserved motifs analysis of EgHMs}

The $109 \mathrm{EgHM}$ genes structural analysis (intron-exon organization) was analyzed by the Gene Structure Display Server (http://gsds.cbi.pku.edu.cn/). The conserved motifs analysis of all identified EgHM proteins were investigated by MEME tool (http:// meme- suite. org/tools/ meme).

\section{EgHM gene duplications, phylogenetic relationships, and their distribution on chromosomes}

We explored the duplications of 109 EgHM gene family members in oil palm genome using the MCScanX tool with default parameters [32, 33]. Further, we mapped the location of all 109 EgHMs across 16 chromosomes of oil palm from the available genome database of oil palm. We also generated the phylogenetic tree for each type (HMT, HDM, HAT \& HDAC) of oil palm HMs with HM genes Arabidopsis and Oryza sativa using MEGA 7.0 [34] by Maximum Likelihood method, with a bootstrap value of 1000 replications.

\section{Plant Materials}

Oil palm (Elaeis guineensis) immature zygotic embryos were selected as explants for oil palm tissue culture and followed the method as described by Silva et al. (2012)[35] with few modifications. The calli (non-embryogenic or embryogenic) was induced from immature zygotic embryos on callus induction media (CIM).The explants were cultured on CIM: (1/2 MS medium supplemented with $30 \mathrm{mg} / \mathrm{l}$ picloram, $100 \mathrm{mg} / \mathrm{l}$ casein hydrolysate, $500 \mathrm{mg} / \mathrm{l} \mathrm{L-glutamine,} 200 \mathrm{mg} / \mathrm{l}$ aspargine, $200 \mathrm{mg} / \mathrm{l}$ arginine, $2 \mathrm{mg} / \mathrm{l}$ glycine, $100 \mathrm{mg} / \mathrm{l}$ adenine sulfate, $100 \mathrm{mg} / \mathrm{l}$ citric acid, $100 \mathrm{mg} / \mathrm{l}$ ascorbic acid, $30,000 \mathrm{mg} / \mathrm{l}$ sucrose, and $3,000 \mathrm{mg} / \mathrm{l}$ Phytagel). The EC and NEC were induced after 3 months of repeated subcultures. The Somatic embryogenesis (SE) was induced by transferring EC to somatic embryo induction medium (SIM: CIM without picloram). The somatic embryo was induced in SIM after four months of culture. The EC, 
NEC, and SEs were collected at their stages and then quickly frozen in liquid nitrogen followed by the storage of $-70^{\circ} \mathrm{C}$ for subsequent RNA extraction.

\section{EgHM s expression analysis from available transcriptome data}

We downloaded the transcriptome data of oil palm somatic embryogenesis stages (EC, NEC, and SE; Accession number: PRJNA699335) from the Sequence Read Archive (SRA) database of NCBI. The heatmap (http://www.omicshare.com/tools) was generated to calculate EgHMs expression levels in three different stages of somatic embryogenesis using RPKM values (RPKM=

$\frac{10^{6} \mathrm{C}}{\text { |raisebox1 ex } \$ \mathrm{NL} \$ / \text { raisebox }-1 \text { ex } \$ 10^{3} \$}$ [32]

\section{EgHM s gene expression analysis by quantitative real-time PCR}

Total RNA was extracted from the different stages of calli (NEC, EC, and SE ) during somatic embryogenesis using RNAprep pure Plant Kit (Tiangen, Beijing, China) by following the manufacturer's instructions. The first-strand cDNA was synthesized using EasyScript ${ }^{\circledR}$ First-Strand cDNA Synthesis SuperMix (TransGen, Beijing, China) kit. The relative expression analysis of the $99 \mathrm{EgHM}$ genes from the 109 identified $H M$ genes was carried out by using the real-time qPCR method. The real-time qPCR was performed with SYBR® Select Master Mix (Thermo Fisher Scientific, Waltham, USA). The ABI QuantStudio $^{\text {TM }} 6$ Flex quantitative real-time PCR instrument (Thermo Fisher, Waltham, USA) was used to analyze the data. The relative expression levels of $99 \mathrm{HM}$ genes at different stages of somatic embryogenesis were calculated via $2^{-\triangle \Delta C t}$ method. The qPCR primers for analyzing the expression of 99 HM genes were designed using QuantPrime qPCR primer designing tool (https:// quant prime. mpimpgolm. mpg. de/) and listed in Supplementary Table.3. All the qPCR reactions were performed with three biological and three technical replications. The oil palm actin gene (EgActin1) was used as an internal control to check the expression of EgHMs. The statistical significance at $\mathrm{p}<0.05$ was determined by using One-Way ANOVA

\section{Results:}

\section{Identification of $\mathrm{HM}$ gene family in oil palm genome}

In this investigation, a total of 109 EgHM gene family members such as 48 of HMTs (histone methyltransferases); 27 of HDMs (histone demethylases); 13 of HATs (histone acetylases), and 21 of HDACs (histone deacetylases) were successfully identified in the oil palm genome via genome-wide analysis. All the EgHM family members are categorized into 11 subfamilies (SDG, PRMT, HDMA, JMJ, HAG, HAM, HAC, HAF, HAD, SRT \& HDT) based on their protein domain architecture. The HMT family is included with 39SDGs and 9PRMTs; HDM family is contained with 3HDMAs and 24JMJs; HAT family is included with 4HAGs, $2 \mathrm{HAMs}, 6 \mathrm{HACs}$, and $1 \mathrm{HAF}$; HDAC family included with 15HADs, 3SRTs, and $3 \mathrm{HDT}$ sub-families. All of the gene IDs of EgHM family members were provided in Supplementary Table 1. The 
pl and Molecular weight of the oil palm HM family members were ranged from 4.57 to 9.95 and 12.3 to 273.3 respectively (Supplementary Table 1 ). The EgHMs are predominantly localized to the cytoplasmic regions. (Supplementary Table 2).

\section{Gene structure and conserved motif analysis of EgHM family members}

We analyzed the gene structure of all EgHM gene family members using the Gene Structure Display Server tool. We found the occurrence of varied numbers (2-34) of exons among the HM gene family members (Fig. 1). The highest number (34) of exons was observed in EgHMT members i.e. EgSDG18 and the least number (2) of exons were observed in EgSDG39(Fig. 1). We found10 conserved motifs among the 109 HM gene family of oil palm(Fig. 2).

\section{Chromosomal distribution of EgHM members in oil palm genome}

The chromosomal distribution of 109 EgHM gene family members was also examined across the 16 chromosomes of the African oil palm genome. The EgHM gene family members were unevenly distributed on the chromosomes(Fig. 3). Among the identified $109 \mathrm{EgHMs}$, only 86 were mapped across the 16 chromosomes(Fig. 3). We have not observed the mapping of $26 \mathrm{EgHM}$ members on any of the chromosomes. Chromosomes 1 and 9 had the highest number (9) of EgHM family genes, whereas chromosome 14 had only one EgHM family gene (Fig. 3). Apart from chromosome 14, all of the remaining chromosomes at least contained 3 or more EgHM family genes.

\section{EgHM gene duplication in oil palm genome}

To know the expansion of the $H M$ gene family in oil palm genome, we generated a gene duplication event diagram for duplicated blocks using a Circos algorithm. In a total, 37 pairs of EgHMs were identified from 16 chromosomes of oil palm, including 14 pairs of EgSDGs; 4 pairs of EgPRMTs; 6 pairs of EgJMJs; 2 pairs of EgHAMs; 1 pair of EgHACs; 9 pairs of EgHDAs, and 1 pair of EgSRTs(Fig. 4). The paired EgHM duplicated genes were all located in different and same chromosome blocks of oil palm genome(Fig. 4). Moreover, Chromosome 1 and Chromosome 6 had 6 and 5 number of duplicated genes respectively. However, chromosome 14 block had no duplicated HM genes(Fig. 4). These results demonstrated the expansion of EgHMgene family that occurred through these duplicated regions.

\section{Phylogenetic analysis between oil palm, rice, and Arabidopsis HM gene family}

To elucidate the evolutionary relationship between oil palm, rice, Arabidopsis, we generated the rooted phylogenetic trees for each HM gene family (HAT, HDAC, HDM, and HMT) (Figs. 5, 6,7 and 8). The phylogenetic tree for subfamilies of each $\mathrm{HM}$ gene family was classified and clustered into diverse trends. The phylogenetic tree of HAT family showed that all the HAG, HAM, HAC, and HAFs of oil palm, rice, and Arabidopsis were not clustered together and they clustered in a species-specific manner and also in mixed type (Fig. 5). For HDAC family, HDTs and SRTs clustered in a species-specific manner, whereas HDAs clustered together (Fig. 6). The phylogenetic tree of HDMs (HDMAs and JMJs) also showed a different trend with a species-specific type of clustering (Fig. 7). The phylogenetic tree of HMT family 
revealed that all the SDGs and PRMTs genes were clustered together (Fig. 8). Altogether, our results indicated that there is a clear evolutionary relationship and diversification between oil palm, rice, and Arabidopsis HM gene family members.

\section{Expression levels of EgHMs in different somatic embryogenic stages (Embryogenic calli, Non- embryogenic calli, and somatic embryos)}

Based on the available transcriptome data of oil palm EC, NEC, and SE (https://www.ncbi.nlm.nih.gov/bioproject/PRJNA699335) stages were downloaded from the NCBI website and analyzed the expression levels of all identified $109 \mathrm{EgHM}$ gene family members. The transcript abundance of EgHMs in various stages of oil palm somatic embryogenesis was analyzed by generating the heatmap with the help of FPKM values. As shown in Fig. 9, EgHM gene members showed differential expression in different stages of somatic embryogenesis of oil palm. However, most of the genes were down-regulated in all three stages of somatic embryogenesis (Fig. 9). The majority of the HMTS (SDGs \& PRMTs) are expressed during EC and NEC stages (Fig. 9). The HDAC gene EgHDT1 is highly expressed in all three stages of somatic embryogenesis, whereas EgHDA15 is highly expressed in the somatic embryo development stage(Fig. 9). Our results elucidated the role of specific EgHMs during the conversion of NEC to SE during oil palm somatic embryogenesis.

\section{Real time-PCR expression analysis of candidate EgHM family genes}

A total of $99 \mathrm{EgHM}$ genes were selected and analyzed their relative expression levels during different stages (NEC, EC \& SE) of somatic embryogenesis of oil palm through q-RTPCR. Our results revealed the varied expression levels of selected $E g H M$ s during various stages of somatic embryogenesis. The relative expression of HMTs (PRMTs \& SDGs) was significantly higher in EC and SE stages than NEC stage(Fig. 10). EgPRMT2 is highly expressed in EC and SE stages, whereas EgPRMT8 has shown the highest expression in SE stages of somatic embryogenesis (Fig. 10). EgPRMT2 \& 5 have shown similar expression in all three stages of somatic embryogenesis. EgSDG24, 28, 35 were highly expressed in SE stage and EgSDG18 has shown the highest level of expression in NEC stage. EgSDG19, 20, and 37 have shown significantly higher expression in EC stage(Fig. 10). The relative expression of HDMs (HDMAs \& $J M J s$ ) was significantly higher in EC and SE stages, whereas EgJMJ20 has shown the highest expression in NEC stage and also in SE stage(Fig. 10). The relative expression of HATs was also significantly higher in both EC and SE stages. Some of them have shown the highest expression in specific stages (either in EC or SE) (Fig. 10). The relative expression of a majority of HDACs is also higher in EC and SE stages than NEC. EgSRT1 and EgHDA15 were highly expressed in SE stage (Fig. 10). Taken together, all these results indicating the potential role of some EgHMs in somatic embryogenesis of oil palm.

\section{Discussion:}

Somatic embryogenesis is an important tissue culture approach for plant regeneration and it incites various epigenetic changes such as histone modifications (methylation, demethylation, acetylation, and 
deacetylation [24, 25]. During the somatic embryogenesis process, various histone modifications are potentially regulated $[24,26]$. The histone modifications activity leads to the altered gene expression of somatic embryogenesis process [24]. These histone modification changes regulated by various genes during somatic embryos potentially affect the development of somatic embryos. It's necessary to know the information of various genes that regulate the histone modifications during somatic embryogenesis. To date, none of the studies provided brief information on histone modification genes of oil palm during somatic embryogenesis process. To the best of our knowledge, this is the first report on the identification and characterization of $H M$ genes in oil palm during somatic embryogenesis process. In our study, we identified a total of $109 \mathrm{HM}$ genes including 48 of HMTs (histone methyltransferases); 27 of HDMs (histone demethylases); 13 of HATs (histone acetylases), and 21 of HDACs (histone deacetylases) through genome-wide analysis and analyzed their expression patterns during different stages (NEC, EC, and SE) of somatic embryogenesis process of oil palm. We reported a comprehensive study including gene structural organization, motif composition, and location on chromosomes, duplication events, and phylogenetic analysis of all of identified 109 EgHMs.

The number (109) of identified HMs in oil palm genome were neither more nor lesser than the identified HM gene family members in other crops including Malus domestica (198 HMs) [8] ; Lycopersicum esculentum (125 HMs) [17] ; in Citrus sinensis (136 HMs) [18]; Litchi chinensis (87 HMs ) [9], indicating that the variance in occurrence of a number of $H M$ genes is not related with the genome size of species. The identified EgHM gene family members were divided into four categories and then 11 sub-families, which is similar to the classification of $H M$ genes published previously in other crops $[8,9,17,18]$. The diversification of the gene family is majorly associated with the intron-exon organization (gain or loss) of genes [27]. Our study also demonstrated that the EgHM genes with similar structural organization were clustered together, whereas others with the different structural organizations were grouped distantly. Our findings have coincided with the previous studies genome-wide identification studies of $H M$ gene families in the apple and citrus genome $[8,18]$. The typical domains in gene clusters of $H M$ gene families are conserved in most of the crops $[8,17,18]$. Moreover, oil palm $H M$ family genes also contained the same typical domain structure as similar to other plant $H M$ gene families $[8,17,18]$.

The phylogenetic analysis of oil palm $H M$ gene family with Arabidopsis and rice was analyzed for each family (HMT, HDM, HAT, and HDAC). They were clustered in species-specific manner which was slightly different from the previous reports on HM gene families in various crops [8, 17]. Our results supporting the diversification of oil palm $H M$ gene family from other crops, however, future phylogenetic studies are needed to know the evolutionary relationship with other closely related palm plants. The chromosomal distribution of all $109 \mathrm{HM}$ genes was uneven across 16 chromosomes of oil palm and our results are coinciding with the previous reports on $H M$ gene family distribution on apple and citrus genome $[8,17]$. Gene duplication plays an essential role in making the complexity of genomes and also in evolutionary lineages $[28,29,30]$. Our results also demonstrated the tandem duplication of EgHMs in oil palm genome during the evolutionary process. These findings are consistent with the expansion of $H M$ gene families during the evolution of the apple genome [8] . 
The epigenetic events trigger the expression of a specific set of genes that are responsible for cellular totipotency [31]. The histone modifications modulate the expression of genes responsible for somatic embryogenesis [26, 31]. In our study, several genes encoding histone methylation, demethylation, histone acetylation, and histone deacetylation were identified through genome-wide study and analyzed their expression analysis during different stages (NEC, EC \& SE) of somatic embryogenesis. In this study, several EgHMs displayed their differential expression between different stages of somatic embryogenesis of oil palm as shown in Fig. 10. The identified EgHMs may provide insightful information to elucidate the molecular mechanisms associated with the somatic embryogenesis of oil palm.

\section{Conclusions:}

In conclusion, this is the first report on genome-wide analysis of histone modification genes (HMT, HDM, $H A T$, and $H D A C$ ) in oil palm. From this study, a total of 109 EgHMs were identified and analyzed candidate genes expression patterns during somatic embryogenesis of oil palm. Moreover, comprehensive information regarding their gene structure, motif, composition, chromosomal distribution, duplication events, evolutionary relationship with rice, and Arabidopsis was also reported. Furthermore, differential expression of various EgHMs at different stages of somatic embryogenesis was also elucidated through real-time PCR analysis indicating their potential involvement during the somatic embryogenesis of oil palm.

\section{Declarations}

\section{Availability of data and materials}

The transcriptome data used in this study are available in the NCBI Sequence Read Archive (SRA) with BioProject accession number PRJNA699335. All data sets analyzed during this research are provided as Supplementary files and also included in the article. The data sets generated during this study are also available from the corresponding author on reasonable request.

Author Contributions: LZ assessed the conceptualization of research. LZ and RY conceived and designed the methodology. LZ, RY, LJ, YY, HC, and ZZ analyzed the data. RY prepared and wrote original manuscript draft supervision. LZ supervised the research. All authors have read and approved.

Funding: This research work was financially supported by the National Natural Science Foundation of China (No: 31870670) and also basic support from the Chinese Academy of Tropical Agricultural Sciences, China.

\section{Ethics approval and consent to participate}

Not applicable.

\section{Consent for publication}


Not applicable.

\section{Competing interests}

The authors declare that they have no competing interest.

\section{References}

1. Zhang X. The epigenetic landscape of plants. Sci 2008, 320:489-492.

2. Klose RJ, Zhang Y. Regulation of histone methylation by demethylimination and demethylation. Nat Rev Mol Cell Biol 2007, 8:307-318.

3. Aquea F, Vega A, Timmermann T., Poupin MJ, Arce-Johnson P. Genome-wide analysis of the SET DOMAIN GROUP family in grapevine. Plant Cell Rep 2011, 30:1087-1097.

4. Hollender C, Liu Z. Histone deacetylase genes in Arabidopsis development. J Int Plant Biol 2008, 50:875-885.

5. Jiang D, Yang W, He Y, Amasino RM. Arabidopsis relatives of the human lysine-specific Demethylase1 repress the expression of FWA and FLOWERING LOCUS C and thus promote the floral transition. Plant Cell 2007, 19:2975-2987.

6. Pandey R, Muller A, Napoli CA, Selinger DA, Pikaard CS, Richards EJ, Bender J, Mount DW, Jorgensen RA. Analysis of histone acetyltransferase and histone deacetylase families of Arabidopsis thaliana suggests functional diversification of chromatin modification among multicellular eukaryotes. Nucleic Acids Research 2002, 30:5036-5055.

7. Feng Q, Wang HB, Ng HH, Erdjument-Bromage H, Tempst P, Struhl K, Zhang Y. Methylation of H3lysine 79 is mediated by a new family of HMTases without a SET domain. Curr Biol 2002, 12:10521058.

8. Fan S, Wang J, Lei C. Identification and characterization of histone modification gene family reveal their critical responses to flower induction in apple. BMC Plant Biol 2018, 18:173. https://doi.org/10.1186/s12870-018-1388-0.

9. Peng M, Ying P, Liu X., Li C, Xia R, Li J, Zhao M. Genome-Wide Identification of Histone Modifiers and Their Expression Patterns during Fruit Abscission in Litchi. Front. Plant Sci 2017, 8, 639.

10. Ahmad A, Cao X. Plant PRMTs broaden the scope of arginine methylation. Journal of Genetics and Genomics 2012, 39:195-208.

11. Dong G, Ma DP, Li J. The histone methyltransferase SDG8 regulates shoot branching in Arabidopsis. Biochem Biophys Res Commun 2008, 373:659-664.

12. Tian L, Fong MP, Wang JJ, Wei NE, Jiang H, Doerge RW, Chen ZJ. Reversible histone acetylation anddeacetylation mediate genome-wide, promoter-dependent and locus-specific changes in gene expression during plant development. Genetics 2005, 169:337-345.

13. Sheldon CC, Finnegan EJ, Dennis ES, Peacock WJ. Quantitative effects of vernalization on FLC and SOC1 expression. Plant Journal 2006, 45:871-883. 
14. Chen ZJ, Tian L. Roles of dynamic and reversible histone acetylation in plant development and polyploidy. Biochim Biophys Acta 2007, 1769:295-307.

15. Ma X, Lv S, Zhang C, Yang C. Histone deacetylases and their functions in plants. Plant Cell Rep 2013, 32:465-478.

16. Wang Z, Cao H, Chen F, Liu Y. The roles of histone acetylation in seed performance and plant development. Plant Physiol Biochem 2014, 84:125-133.

17. Cigliano RA, Sanseverino W, Cremona G, Ercolano MR., Conicella C, Consiglio FM. Genome-wide analysis of histone modifiers in tomato: gaining an insight into their developmental roles. BMC Genomics 2013, 14:57.

18. Xu J, Xu H, Liu Y, Wang X, Xu Q, Deng X. Genome-wide identification of sweet orange (Citrus sinensis) histone modification gene families and their expression analysis during the fruit development and fruit-blue mold infection process. Front Plant Sci 2015, 6:607.

19. Barcelos E, Rios SA, Cunha RNV, Lopes R, Motoike SY, Babiychuk E, Skirycz A, Kushnir S. Oil palm natural diversity and the potential for yield improvement. Front. Plant Sci 2015, 6:190. doi: 10.3389/fpls.2015.00190.

20. Corley RHV. How much palm oil do we need? Environ. Sci. Policy 2019, 12:134-139. doi: 10.1016/j.envsci.2008.10.011.

21. Elhiti M, Stasolla C. Somatic embryogenesis: the molecular network regulating embryo formation. Somatic embryogenesis in ornamentals and its applications. Springer, Berlin 2016, 12:217-229.

22. Smertenko A, Bozhkov PV. Somatic embryogenesis: life and death processes during apical-basal patterning. J Exp Bot 2014, 65:1343-1360.

23. Wang QM, Wang L. An evolutionary view of plant tissue culture: somaclonal variation and selection. Plant Cell Rep 2012, 31:1535-1547.

24. Li HL, Guo D, Zhu JH, Wang Y, Peng SQ. Identification of histone methylation modifiers and their expression patterns during somatic embryogenesis in Hevea brasiliensis. Genetics and molecular biology 2020, 43(1):e20180141. https://doi.org/10.1590/ 1678-4685-GMB-2018-0141.

25. Yakovlev IA, Carneros E, Lee Y. Transcriptional profiling of epigenetic regulators in somatic embryos during temperature induced formation of an epigenetic memory in Norway spruce. Planta.2016, 243:1237-1249.

26. De-la-Peña C, Nic-Can GI, Galaz-Ávalos RM, Avilez-Montalvo R, Loyola-Vargas VM. The role of chromatin modifications in somatic embryogenesis in plants. Front Plant Sci 2015, 6:635.

27. Xu GX, Guo CC, Shan HY, Kong HZ. Divergence of duplicate genes in exon-intron structure. P Natl Acad Sci USA 2012, 109(4):1187-1192.

28. Si W, Hang T, Guo M, Chen Z, Liang Q, Gu L, Ding T. Whole-genome and transposed duplication contributes to the expansion and diversification of TLC genes in maize. Int J Mol Sci 2019, 20(21):5484. 
29. Flagel LE, Wendel JF. Gene duplication and evolutionary novelty in plants. New Phytol 2009, 183(3):557-564.

30. Moore RC, Purugganan MD. The evolutionary dynamics of plant duplicate genes. Curr Opin Plant Bio 2005, I8(2):122-128.

31. Pasternak T, Dudits D. Epigenetic Clues to Better Understanding of the Asexual Embryogenesis in planta and in vitro. Front. Plant Sci 2019, 10:778. doi:10.3389/fpls.2019. 00778.

32. Zhou LX, Yarra R. Genome-wide identification and expression analysis of bZIP transcription factors in oil palm (Elaeis guineensis Jacq.) under abiotic stress. Protoplasma 2021, Jul 1. https://doi: 10.1007/s00709-021-01666-6.

33. Wang Y, Tang H, DeBarry JD, Tan X, Li J, Wang X, Lee T, Jin H, Marler B, Guo H, Kissinger JC, Paterson AH. MCScanX: a toolkit for detection and evolutionary analysis of gene synteny and collinearity. Nucl. Acids Res 2012, e49.

34. Kumar S, Stecher G, Tamura K. MEGA7: molecular evolutionary genetics analysis version 7.0 for bigger datasets. Mol Biol Evol 2016, 33:1870-1874.

35. Silva RC, Luis ZG, Scherwinski-Pereira JE. Differential responses to somatic embryogenesis of different genotypes of Brazilian oil palm (Elaeis guineensis Jacq.). Plant Cell Tiss Org Cult. 2012, 111:59-67.

\section{Figures}




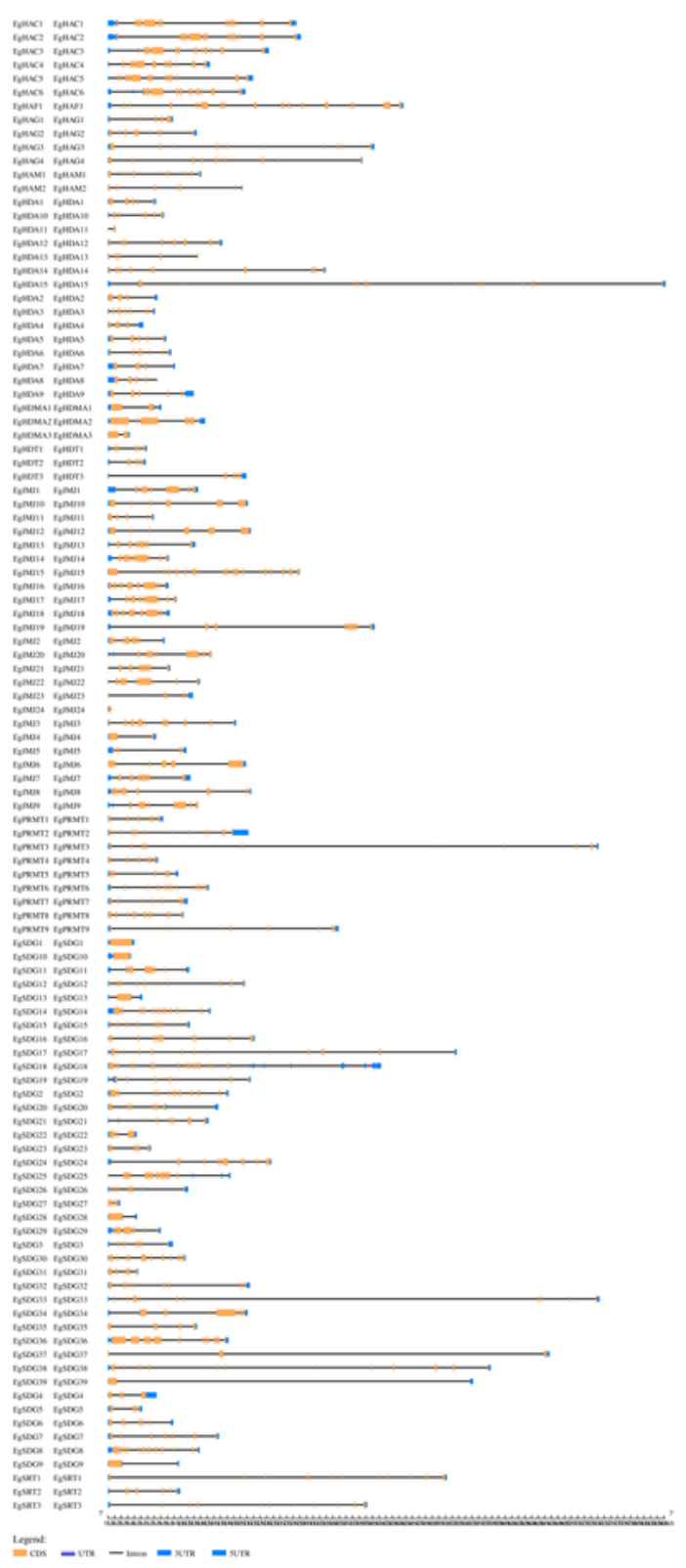

\section{Figure 1}

Gene structural organization of 109 EgHMs. Coding sequences (CDS) are represented by orange color blocks; 3' \& 5' UTRs are regions are represented by blue color blocks; intron regions are represented by black color blocks in the diagram. 

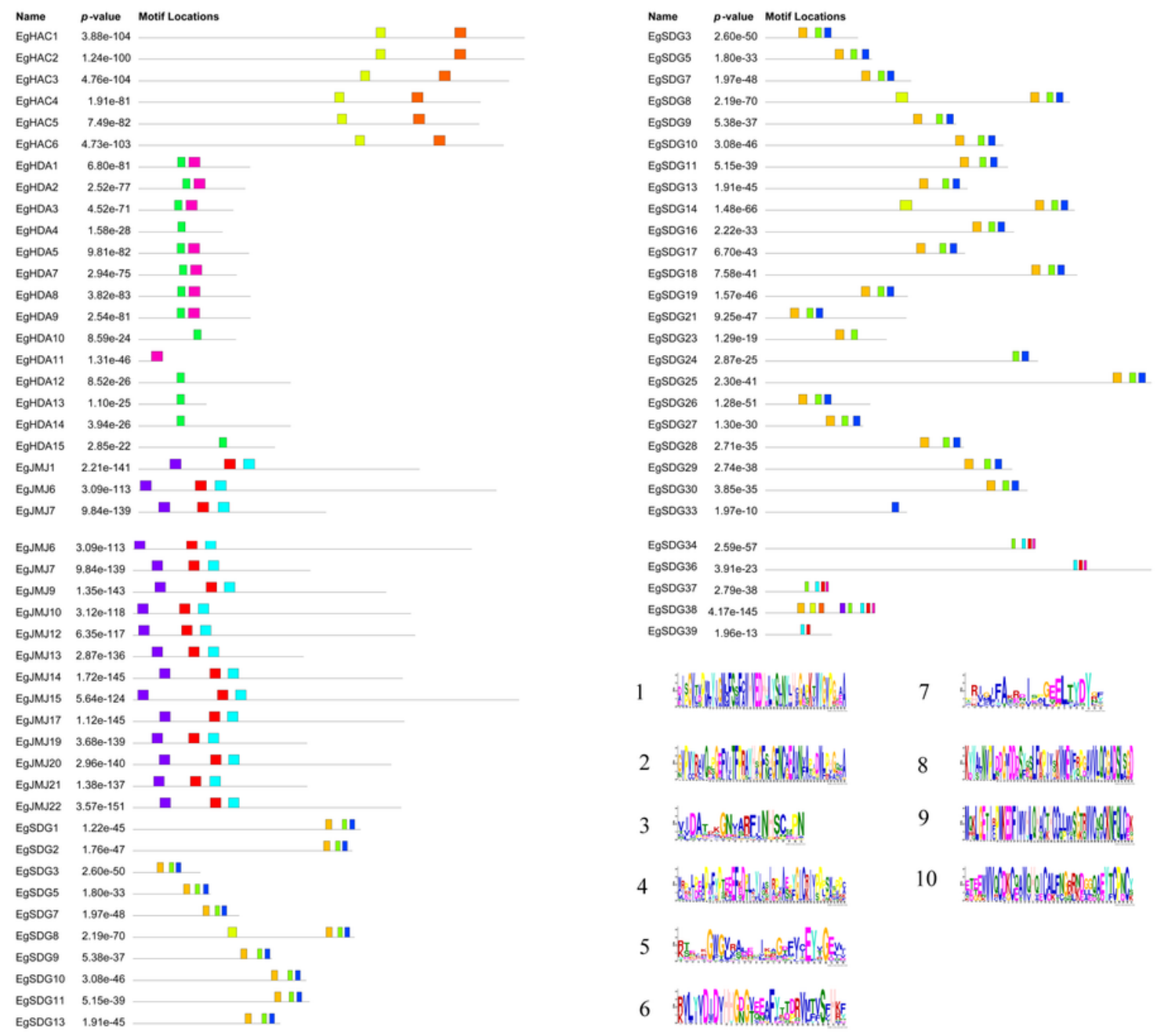

\section{Figure 2}

Occurrence of conserved motifs in EgHM proteins. The MEME tool was used to identify the ten numbers of motifs in EgHM proteins. Each motif in EgHM proteins has shown with different colors. The sequence logo of each motif represents the abundance of each amino acid in their motifs. 


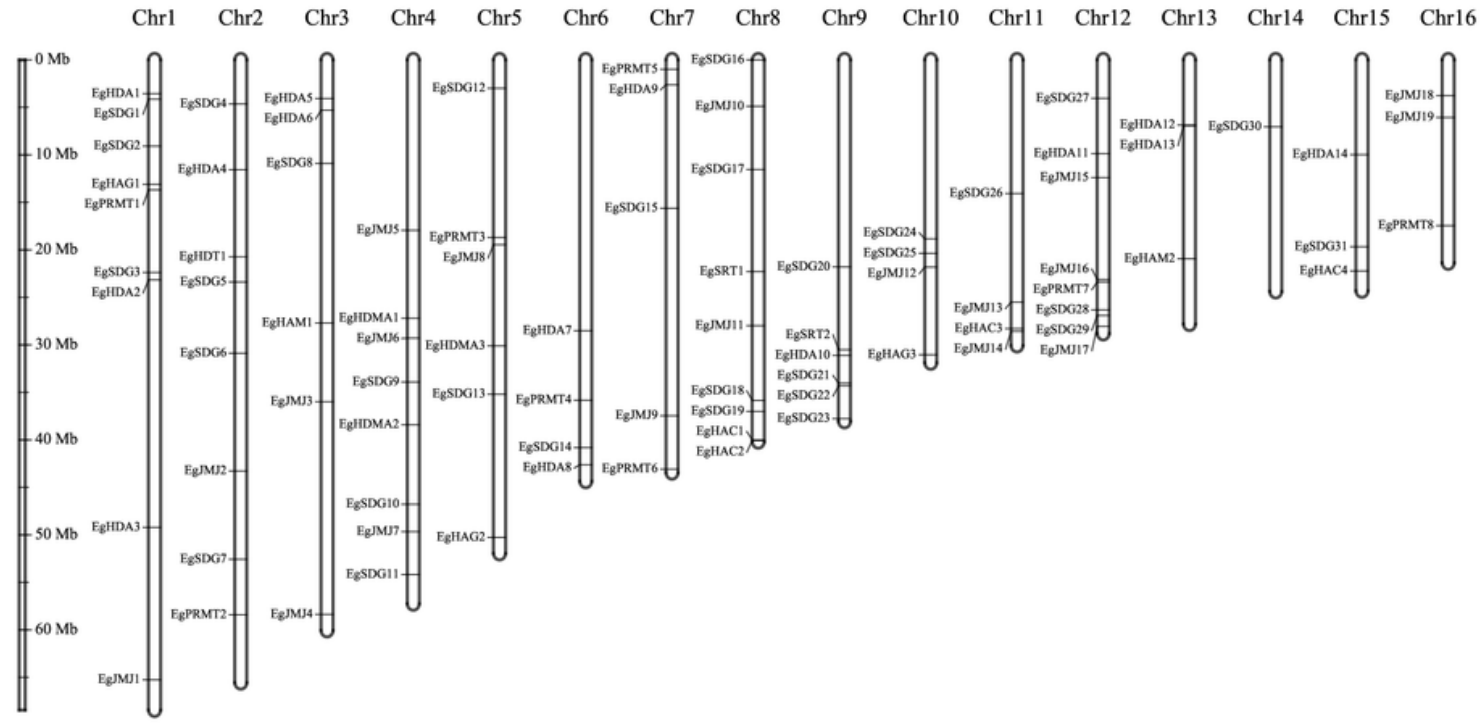

Figure 3

Distribution of EgHMs across 16 chromosomes of oil palm genome. The vertical greyscale on the left side represents the length of the oil palm chromosomes. Gene duplication events of EgHM gene family 


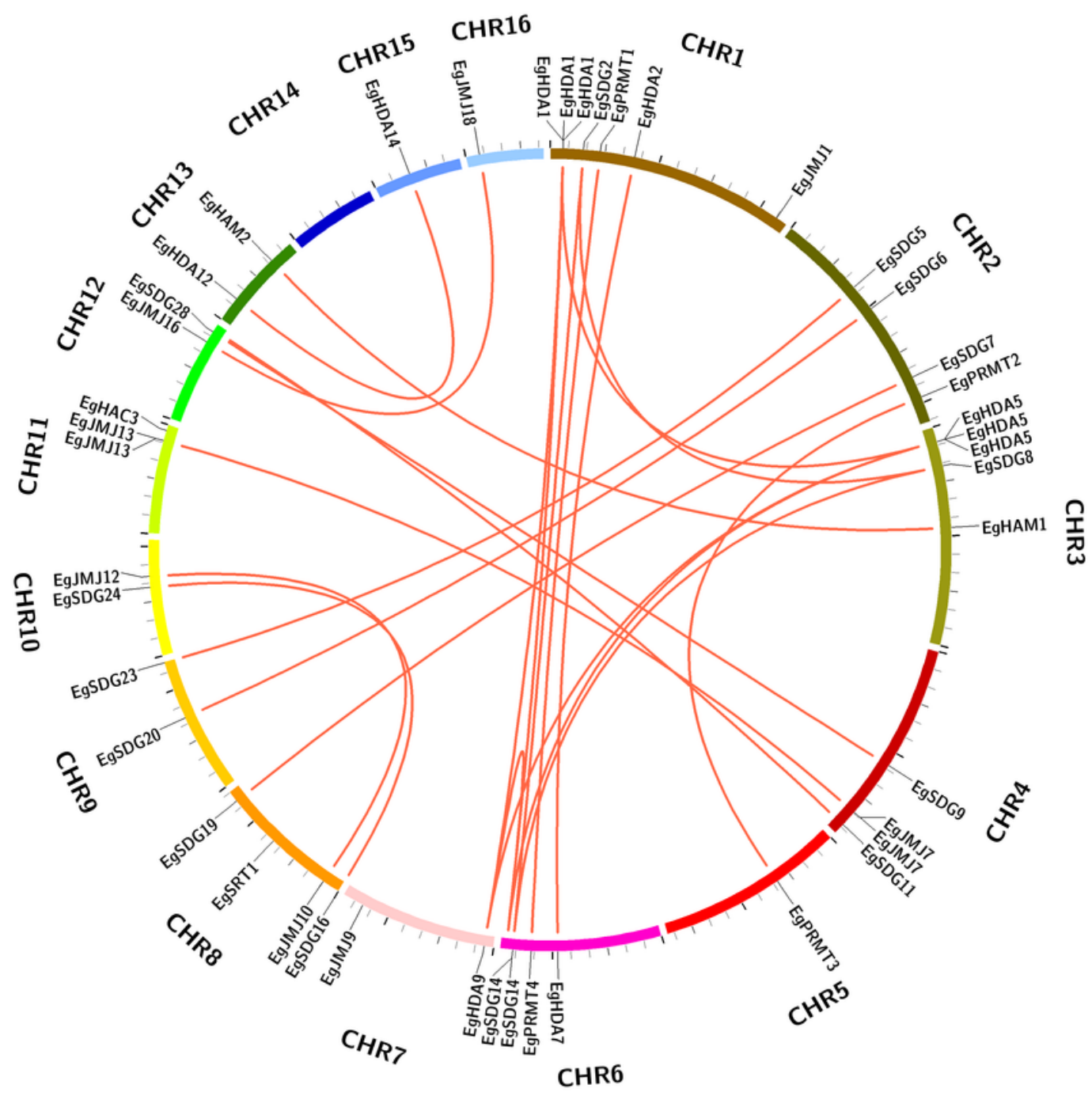

Figure 4

EgHM gene family duplication in oil palm genome. The duplicated EgHM gene pairs are represented with red lines inside the circle view 


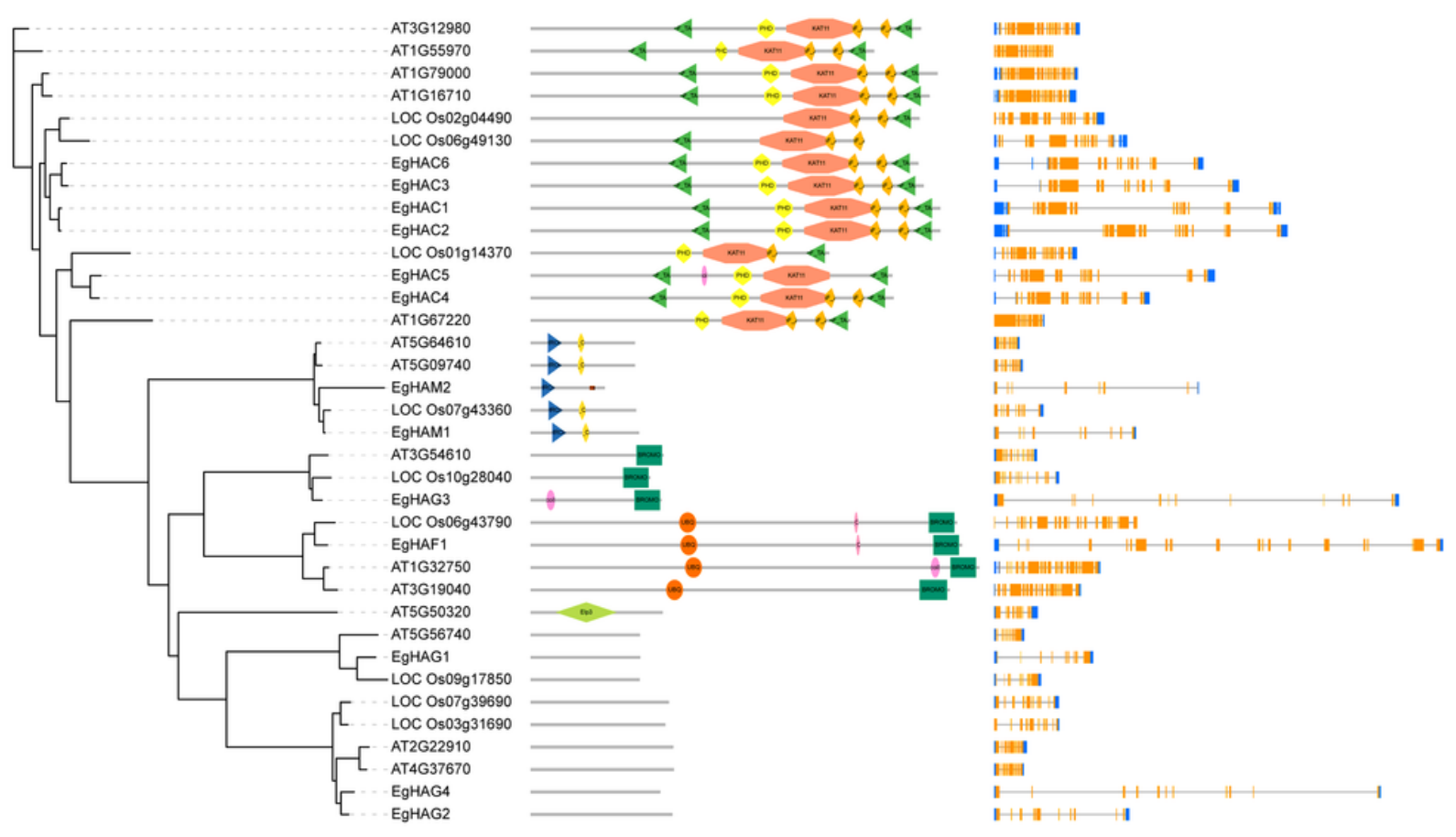

Figure 5

Phylogenetic analysis of EgHAT family with rice and Arabidopsis HAT family genes 


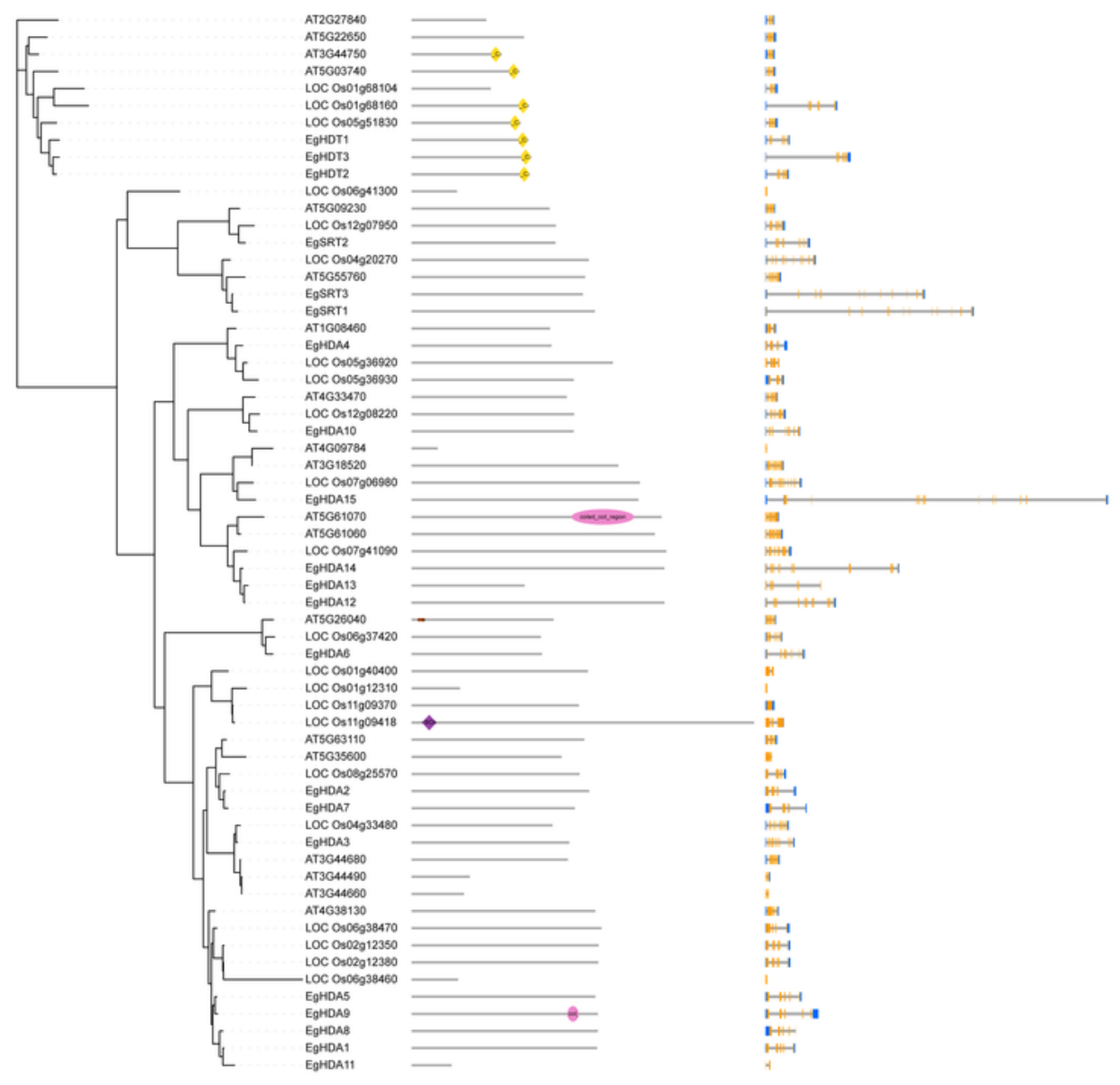

Figure 6

Phylogenetic analysis of EgHDACs with rice and Arabidposis HDAC genes 


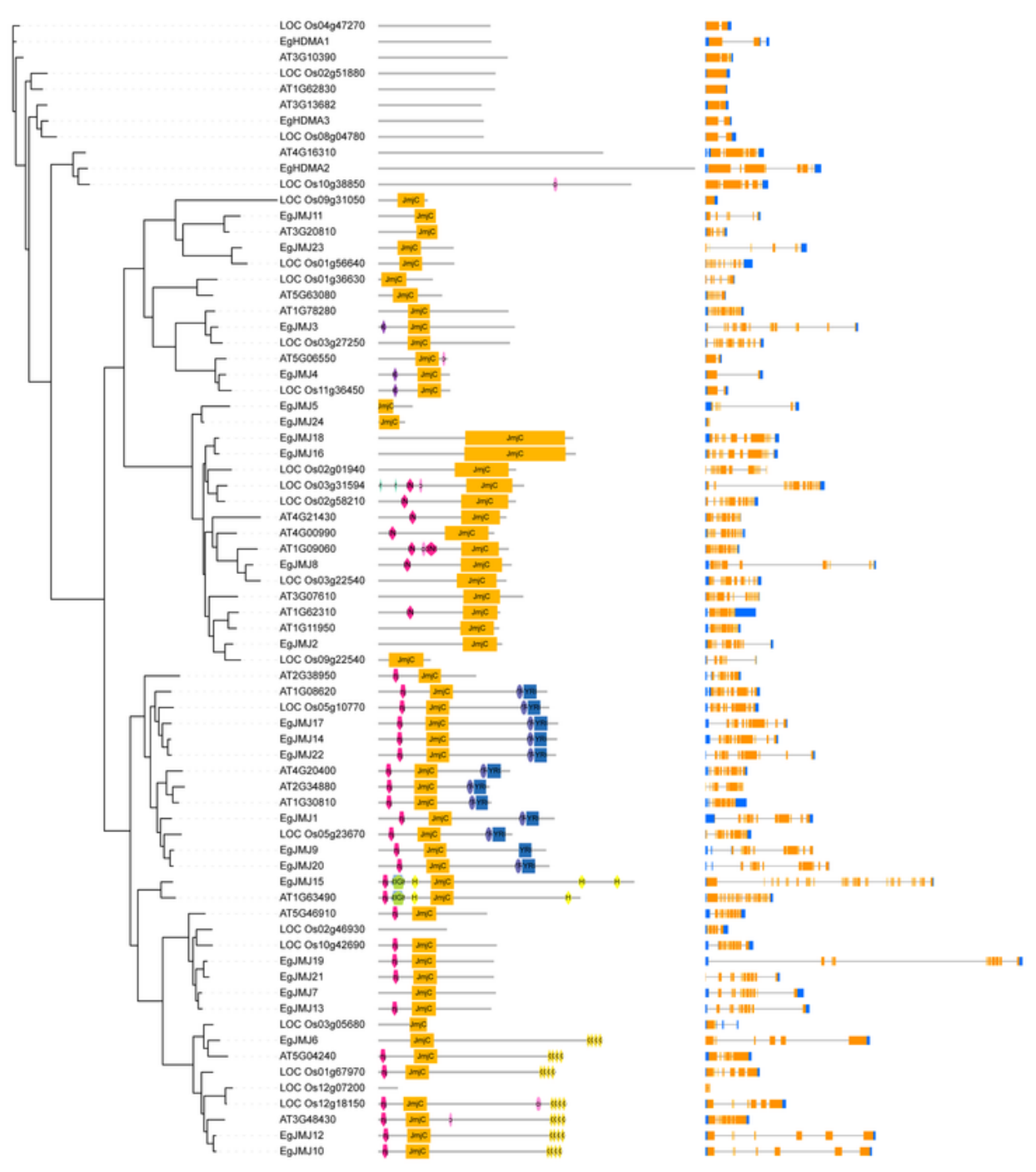

Figure 7

Phylogenetic analysis of EgHDMs with rice and Arabidposis HDM genes 


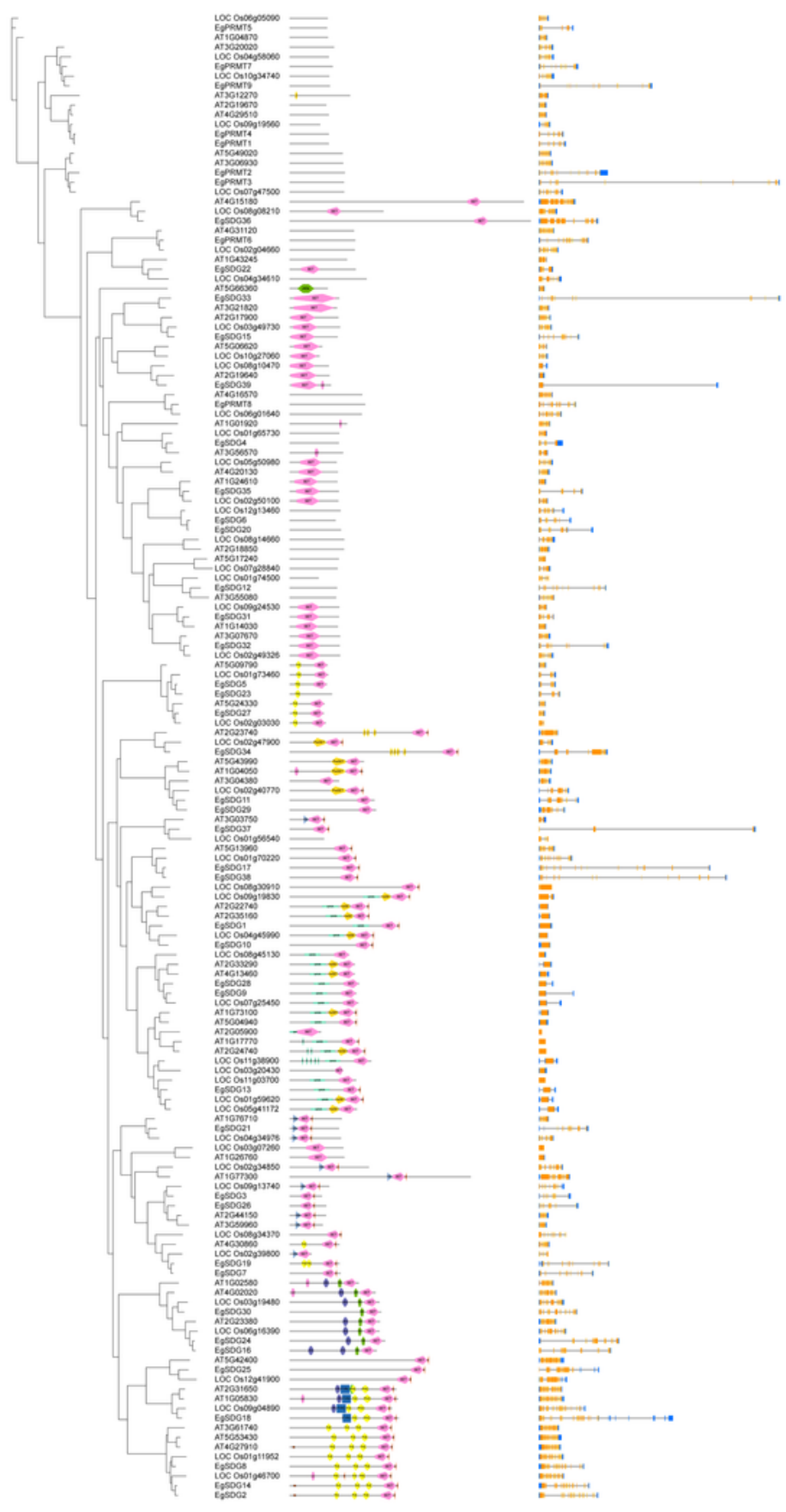

Figure 8

Phylogenetic analysis of EgHMTs with rice and Arabidopsis HMT genes 


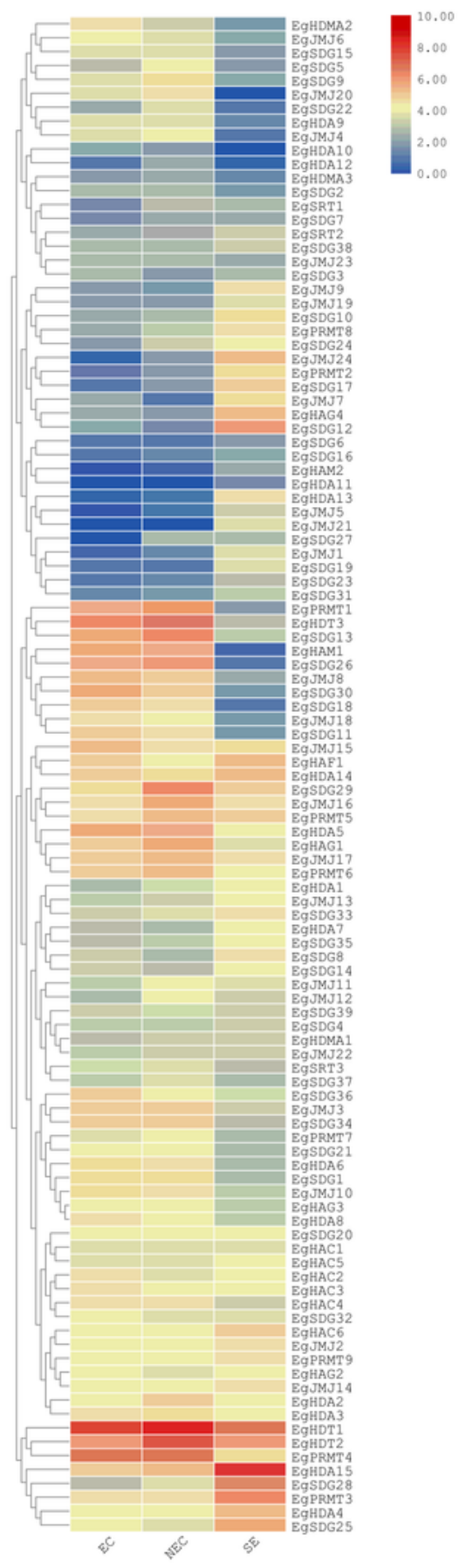

\section{Figure 9}

Heatmap for 109 EgHMs expressions in various stages (Embryogenic CALLI, Non-embryogenic calli, Somatic embryos) of somatic embryogenesis. The color scale indicates EgHMs expression levels. The transcriptome data accession number is PRJNA699335 


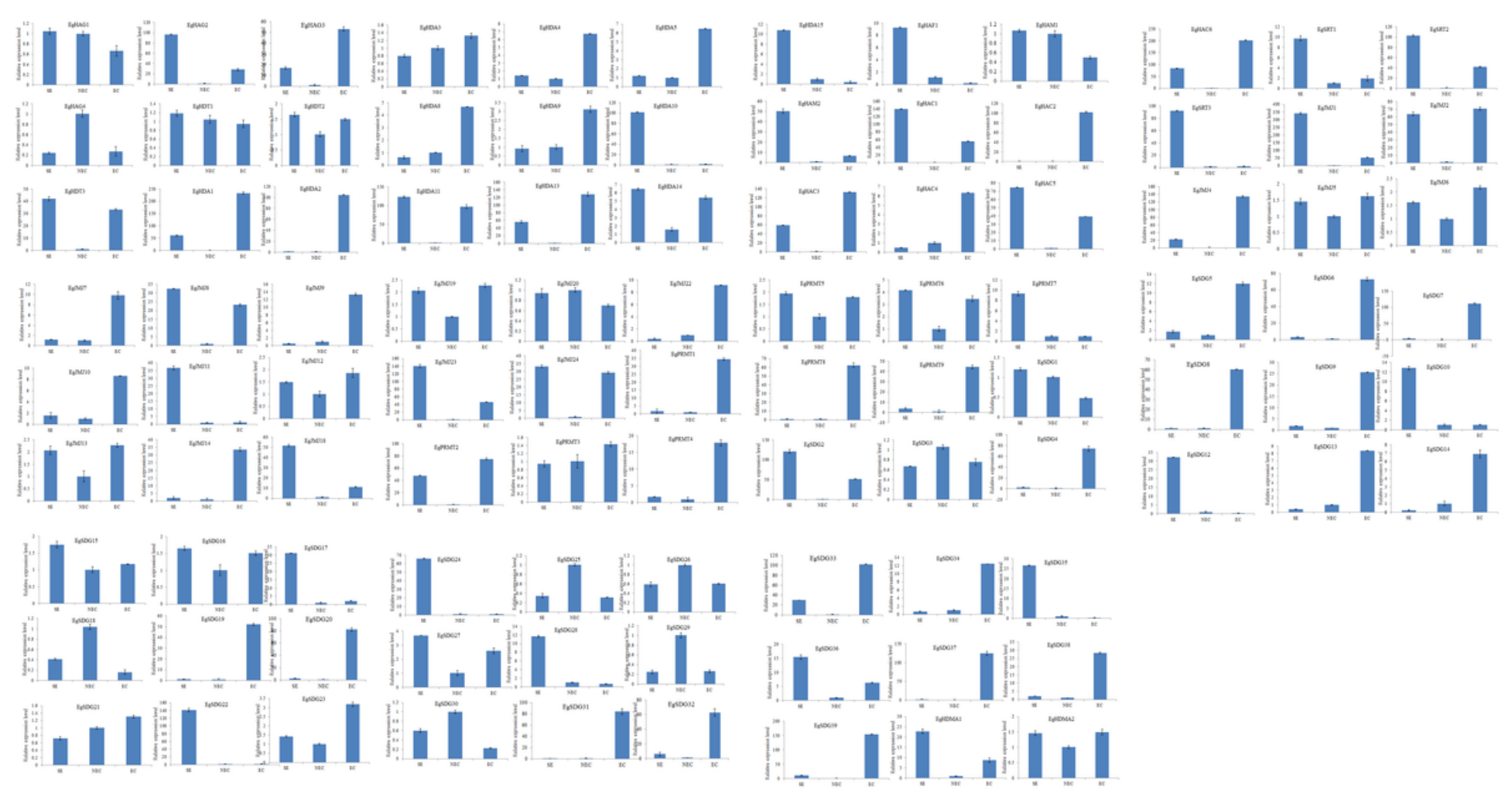

Figure 10

Relative expression analysis of $99 \mathrm{EgHM}$ candidate genes in various stages of somatic embryogenesis in oil palm. The X-axis represents different stages (SE, Somatic embryos; NEC, non-embryogenic calli; EC, embryogenic calli); $Y$-axis represents relative expression of specific EgHM gene. Data represent the mean $\pm \mathrm{SE}$ of three replicates. Asterisks represent significant differences at $\mathrm{P} \leq 0.05\left(^{(}\right)$and $\left.\mathrm{P} \leq 0.01{ }^{(\star *}\right)$.

\section{Supplementary Files}

This is a list of supplementary files associated with this preprint. Click to download.

- SupplemnetaryTable1.xlsx

- SupplementaryTable2.xlsx

- SupplemnetaryTable3.xlsx 